\title{
Timely stereotactic body radiotherapy (SBRT) for spine metastases using a rapidly deployable automated planning algorithm
}

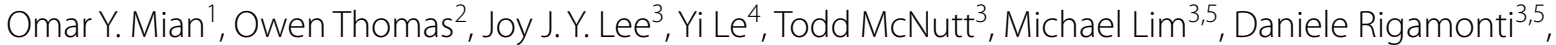 \\ Jean-Paul Wolinsky ${ }^{5}$, Daniel M. Sciubba ${ }^{5}$, Ziya L. Gokaslan ${ }^{5}$, Kristin Redmond $^{3}$ and Lawrence Kleinberg ${ }^{3 *}$
}

\begin{abstract}
Purpose/objectives: The complex planning and quality assurance required for spine SBRT are a barrier to implementation in time-sensitive or limited resource clinical situations. We developed and validated an automated inverse planning algorithm designed to streamline planning and allow rapid delivery of conformal single fraction spine SBRT using widely available technology.

Materials/methods: The Rapid Spine (RaSp) automated script successfully generated single fraction SBRT plans for fourteen complex spinal lesions previously treated at a single high-volume institution. Automated RaSp plans were limited to 5 beams with a total of 15 segments (allowing calculation-based verification) and optimized based on RTOG 0631 objectives. Standard single fraction (16 Gy) stereotactic IMRT plans were generated for the same set of complex spinal lesions and used for comparison. A conservative $2 \mathrm{~mm}$ posterior isocenter shift was used to simulate minor set-up error.
\end{abstract}

Results: Automated plans were generated in under 5 min from target definition and had a mean dose to the PTV of 1663 cGy (SD 131.5), a dose to $90 \%$ of PTV (D90) of 1358 cGy (SD 111.0), and a maximum point dose (Dmax) to the PTV of 2055 cGy (SD 195.2) on average. IMRT plans took longer to generate but yielded more favorable dose escalation with a mean dose to the PTV of 1891 cGy (SD 117.6), D90 of 1731 cGy (SD 126.5), and Dmax of 2218 cGy (SD 195.7). A 2 mm posterior shift resulted in a $20 \%$ (SD $10.5 \%$ increase in cord dose for IMRT plans and a $10 \%$ (SD $5.3 \%$ ) increase for RaSp plans. The $2 \mathrm{~mm}$ perturbation caused 3 cord dose violations for the IMRT plans and 1 violation for corresponding RaSp plans.

Conclusion: The Rapid Spine plan method yields timely and dosimetrically reasonable SBRT plans which meet RTOG 0631 objectives and are suitable for rapid yet robust pretreatment quality assurance followed by expedited treatment delivery. RaSp plans reduce the tradeoff between rapid treatment and optimal dosimetry in urgent cases and limited resource situations.

Keywords: Automated planning, SBRT, Radiation therapy, Spinal lesions

\section{Background}

Spine metastases are a common complication of malignancy, leading to diminished performance status, pain, and neurologic compromise. Radiotherapy is the standard for

\footnotetext{
*Correspondence: kleinla@jhmi.edu

${ }^{3}$ Department of Radiation Oncology and Molecular Radiation Sciences,

Sidney Kimmel Comprehensive Cancer Center, The Johns Hopkins

University School of Medicine, Baltimore, MD, USA

Full list of author information is available at the end of the article
}

non-invasive management of spine metastases in patients without mechanical instability or symptomatic spinal cord compression. Single fraction conventional treatments such as 800 cGy have been shown to be equivalent to fractionated treatment (e.g., 3000 cGy in 10 fractions) in providing pain relief from metastatic disease of the spine without dose limiting or late complications such as myelopathy (Hartsell et al. 2005; Howell et al. 2013). Nevertheless, as many as $60 \%$ of patients treated with conventional spine 
radiotherapy respond inadequately to treatment and retreatment is common. Increasingly conformal radiotherapy methods employing stereotactic set-up have allowed precise and safe delivery of higher doses of radiation to the tumor volume with durable local control rates approaching $90 \%$ (Gerszten et al. 2007). A growing body of clinical data suggests stereotactic body radiation therapy (SBRT) is well tolerated and provides rapid and durable pain relief (Ryu et al. 2011; Amdur et al. 2009; Schipani et al. 2012). SBRT has the added benefit of shortened overall treatment course and smaller treatment volumes with less radiation exposure to adjacent normal tissues, potentially maximizing access to the systemic therapies important in this group of patients. Recent prospective trials including an ongoing cooperative group study (RTOG 0631) are further investigating whether clinical outcome measures such as pain control are improved with stereotactic radiotherapy compared to conventional radiation therapy.

In general, intensity modulated radiotherapy (IMRT) involves inverse planning based on user-defined dosimetric constraints and relies on more complex and dynamic segmentation of beams compared to other conformal techniques, such as forward planned 3D conformal radiotherapy. While other methods utilize multi-leaf collimator beam shaping and can employ stereotactic immobilization, IMRT plans are generally thought to produce more conformal therapeutic volumes by iterative, computation-intensive inverse planning. There are, however, several practical disadvantages to the use of IMRT based stereotactic treatments for spine metastases. In resource limited situations, the availability of trained dosimetrists, planning systems, and linear accelerators equipped to perform IMRT based stereotactic therapy are limited. When available, the complex inverse planning and requirement for IMRT patient-specific quality assurance (QA) can delay the start of treatment and limit the utility of IMRT in the urgent setting. In the appropriate scenario, calculation-based verification is sufficient and is considered both standard and robust (Lo et al. 2013). In addition, the steep dose gradients with highly conformal treatments require labor-intensive stereotactic treatment set-up and verification which can lengthen the treatment time, limit compliance and feasibility in patients with severe tumor-related pain, and increase cost and resource requirements. With these limitations to IMRT in mind, alternative strategies for the rapid and safe delivery of conformal single fraction radiotherapy are desirable. Although planning systems designed to speed the process have recently become commercially available, such resources are not widely available in the United States and worldwide. The present work explores the use of an automated inverse planning workflow (Rapid Spine) designed to minimize planning time, generate rapid high quality plans with limited beam segmentation, and utilize non-IMRT calculation-based verification while adhering to RTOG spine SBRT guidelines and dosimetric constraints.

\section{Methods \\ Patient selection and target lesions}

Fourteen spinal lesions previously treated at a high volume institution with either conventional or stereotactic radiation therapy had new single fraction treatment plans automatically generated using the Pinnacle Planning System and automating scripts. The scripts used in planning are available from the corresponding author. All patients were simulated using a non-contrast enhanced CT scan in the supine position with standard immobilization techniques. Twelve lesions abutted the thecal sac, eight involved spinal pedicles, seven demonstrated epidural extension, and two were circumferential. It is worth noting that controversy exists as to the optimal management in cases of epidural extension in light of data supporting downstaging with minimally invasive separation surgery prior to SBRT (Laufer et al. 2013). In the present study, three patients had undergone debulking surgery, one patient underwent pretreatment vertebroplasty, and the remainder were not considered candidates for surgical decompression. All patients included had disease that was considered clinically urgent. Eight tumors were thoracic, five were lumbar, and one was cervical. Details including histology, spinal column level, planning target volume (PTV) volume, associated symptoms, and lesion characteristics are given in Table 1.

\section{Rapid Spine (RaSp) script}

An automated inverse planning script was developed using the Pinnacle Planning System with the goal of yielding a robust algorithm producing systematized treatment plans applicable to varied and complex cases with minimal need for user individualization. Parameters for the script included the delivery of stereotactic doses to the PTV up to the RTOG 0631 standard of $16 \mathrm{~Gy}$ in a single fraction. The spinal cord was contoured starting $10 \mathrm{~cm}$ above the superior extent of the PTV to $10 \mathrm{~cm}$ below the inferior extent of the PTV. If necessary, the algorithm limited the prescription dose to meet spinal cord constraints as described further below. The clinical target volume (CTV) was manually defined by the treating physician according to international consortium guidelines (Cox et al. 2012). For a given plan, an automated script automatically placed the isocenter at the calculated volumetric centroid point of the target volume, generated a PTV ( $2 \mathrm{~mm}$ uniform expansion of the CTV, excluding the thecal sac), concentric ring ROIs for planning, and a truncated thecal sac (limited 
Table 1 Histology and lesion characteristics

\begin{tabular}{|c|c|c|c|c|c|c|}
\hline ID & Diagnosis & Level & Lesion characteristics & Circumferential & Symptoms & Volume CTV (cc) \\
\hline 1 & Spindle cell & T8 & Epidural extension, compression, T2 Cord changes & No & Pain, Non focal & 29.192 \\
\hline 2 & $\begin{array}{l}\text { Esophageal } \\
\text { Adeno }\end{array}$ & L3 & Wedge deformity, no canal compromise & No & Pain, Non focal & 41.86 \\
\hline 3 & Chordoma & L2 & Pedicle involvement, Mass effect on thecal sac & Pedicle & Pain, Non focal & 82.36 \\
\hline 4 & Adrenal & L3 & Circumferential PTV, debulking of $L 3$, vertebrectomy & Yes & Pain, Non focal & 182.8 \\
\hline 5 & Prostate & L5 & Retropulsion with canal narrowing, pedicle involvement & Pedicle & Pain, urostomy & 68.14 \\
\hline 6 & Breast & $\mathrm{T} 12$ & Expansile s/p vertebroplasty, pedicle but no canal & Pedicle & Pain & 46.16 \\
\hline 7 & Pancreatic & T8-9 & Circumferential, T9 compression, epidural extension & Yes & Pain & 129.932 \\
\hline 8 & Glottic SCC & $\mathrm{T} 2$ & s/p Laminectomy from T2-T4 & No & Pain, ataxia & 5.87 \\
\hline 9 & Melanoma & L5 & b/l Pedicles with epidural extension & Pedicle & Pain, proprioception & 37.75 \\
\hline 10 & Renal cell & T8 & s/p Resection & Pedicle & Pain & 137.61 \\
\hline 11 & Colon & $\mathrm{T} 12$ & Extension into central canal with cord displacement & Pedicle & Pain & 63.57 \\
\hline 12 & Renal cell & T8 & Posterior pedicle involvement and flattening of cord & Pedicle & Pain & 26.36 \\
\hline 13 & Renal cell & C7 & No pedicle, no epidural extension & No & Pain & 16.84 \\
\hline 14 & Renal cell & T3 & Pedicle involvement & Pedicle & Pain & 27.06 \\
\hline
\end{tabular}

to the superior/inferior dimensions of the PTV). A second sequential script placed either 2, 3, 5, or 7 coplanar beams, which were limited to 3 or fewer segments per beam. The same script then optimized the treatment plan in inverse fashion based on fixed treatment objectives following RTOG 0631 protocol for dose specifications, target coverage, cord dose limits (Fig. 1). For each patient, the final plan was optimized: first, a plan was generated using a conservative objective of a point dose maximum to the thecal sac of $12 \mathrm{~Gy}$; if this plan did not meet cord constraints, then an alternate plan using an objective of $10 \%$ of the truncated thecal sac receiving less than 10 Gy was generated. Both plans were evaluated for adherence to treatment objectives. If the objectives on the thecal sac were not met by inverse planning, the prescription dose was lowered to meet these objectives. A hard constraint

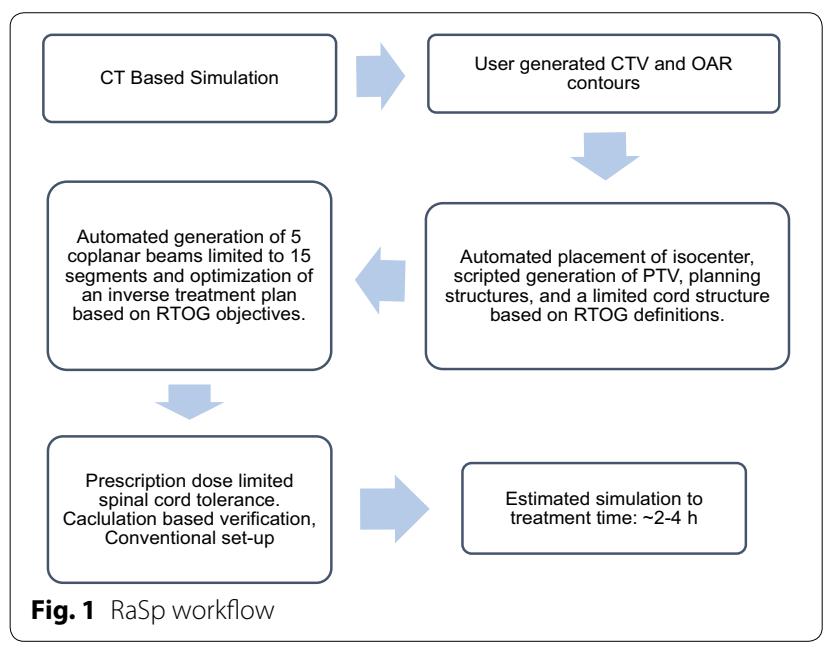

was placed on cord dose so as not to exceed a point maximum of 12 Gy with a dose to $10 \%$ of a limited thecal sac structure (as defined above) of less than $10 \mathrm{~Gy}$.

\section{In silico simulation of setup error}

A $2 \mathrm{~mm}$ anterior/posterior shift was manually introduced by moving the isocenter posteriorly toward the spinal cord to simulate a 'worst case scenario' set-up error. A $2 \mathrm{~mm}$ error was chosen to represent the realistic possibility that set-up errors of this small magnitude may routinely occur and go undetected, particularly when standard, non-stereotactic immobilization is used-a likelihood that is increased in cases where urgent therapy is required. Dosimetric endpoints including CTV coverage and cord dose were obtained before and after this shift and compared to determine the impact of setup error on both IMRT as well as scripted RaSp treatment plans. The intended treatment linear accelerator employed a multi-leaf collimator (MLC) leaf size between 5 and $10 \mathrm{~mm}$.

\section{Results}

Fourteen spinal lesions were examined with a mean CTV volume of 63.97 cc (range 5.87-182.8). The CTV included a single vertebral level for all but one patient, where two vertebral levels were included. Histology and lesion characteristics varied (Table 1). Single fraction IMRT (16 Gy) and 2-, 3-, 5-, and 7-field RaSp automated plans were generated for each lesion to cover the target volume as described in the "Methods" section. For automated RaSp plans, increasing beam number resulted in more conformal automated plans: the 5-field plans qualitatively yielded the best balance between coverage and 

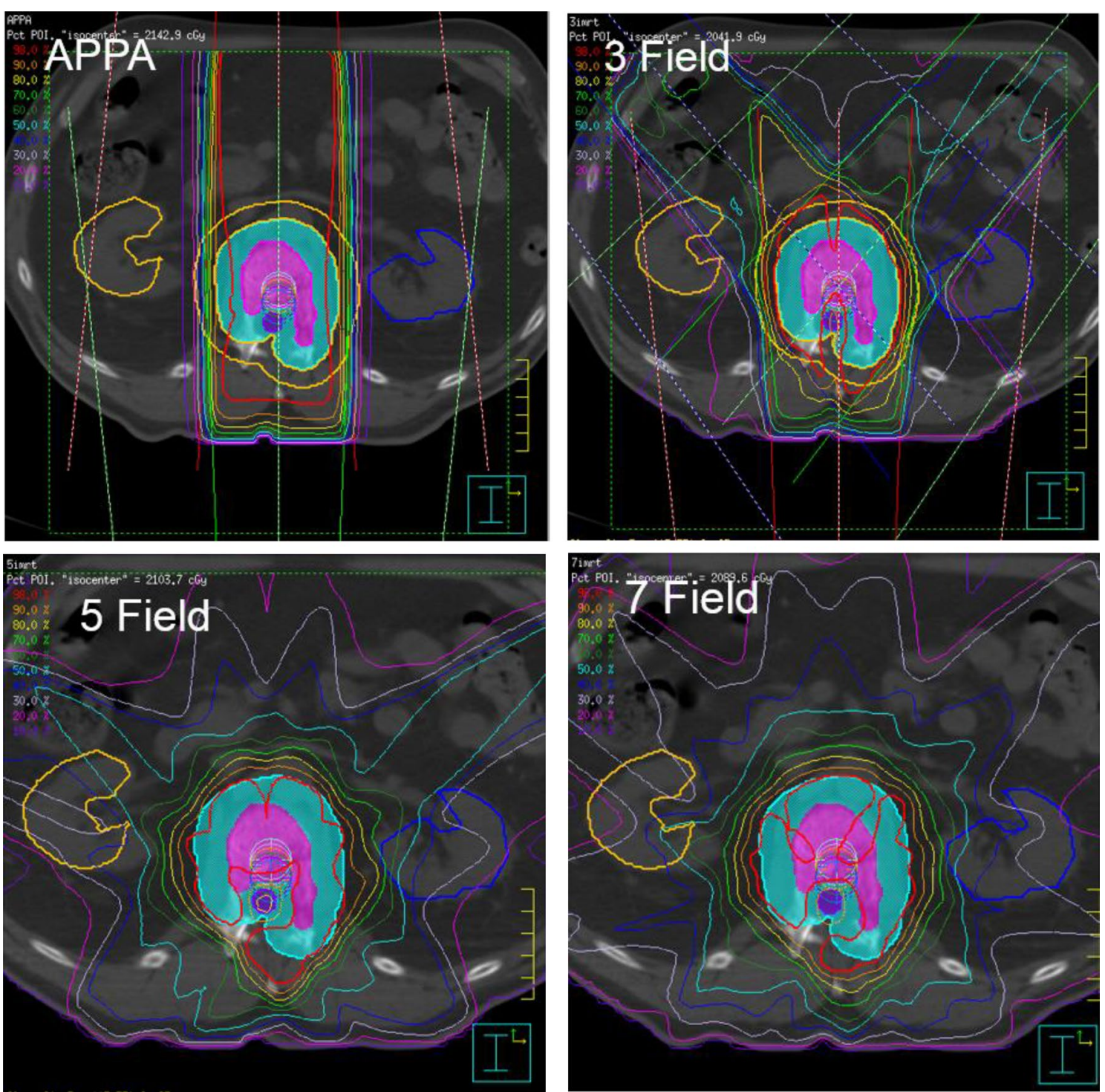

Fig. 2 Representative plan images comparing 2-, 3-, 5-, and 7-field RaSp plans

plan simplicity and robustness (Fig. 2). 5-field plans, limited to 3 segments or fewer per beam, were used for subsequent comparisons with corresponding IMRT plans. All automated plans were generated and optimized in under $5 \mathrm{~min}$. In comparison, IMRT plans took longer to generate, as planning varied from 2 to $4 \mathrm{~h}$. For complex lesions, IMRT plans often took more than $4 \mathrm{~h}$ to complete multiple iterations during plan optimization. In addition, IMRT plans had a higher average beam number, and higher total segment count (7.1 [SD 1.0] beams and 52.6 [SD 11.7] segments).

RaSp plans yielded a mean dose to the PTV of 1663 cGy (SD 131.5, $95 \%$ CI 1582.2-1745.1), dose to $90 \%$ of the PTV (D90) of 1358 cGy (SD 111.0, 95 \% CI 1289.21426.8), and a maximum point dose (Dmax) to the PTV of 2055 cGy (SD 195.2, 95 \% CI 1934.2-2176.3), on average. IMRT plans yielded more favorable dose escalation with a mean dose to the PTV of 1891 cGy (SD 117.6, $95 \%$ CI 1818.3-1964.1), D90 of 1731 cGy (SD 126.5, $95 \%$ CI 1652.5-1809.5), and Dmax of 2218 cGy (SD 195.7, 95 \% CI 2097.1-2339.7) (Fig. 3). Mean point minimum doses were lower but not significantly different between RaSp (846.7 cGy, 95 \% CI 715.3-978.1) and IMRT plans (1035.7 cGy, 95 \% CI 875.2-1196.3).

All initial RaSp and IMRT plans met RTOG 0631 spinal cord limits. As stipulated in 0631, the spinal cord dose remained below 10 Gy to $10 \%$ of the partial spinal cord volume defined as 5-6 $\mathrm{mm}$ above and below the target. In no case was the dose of $10 \mathrm{~Gy}$ exceeded for a spinal cord volume of $0.35 \mathrm{cc}$. The absolute maximum dose to the 

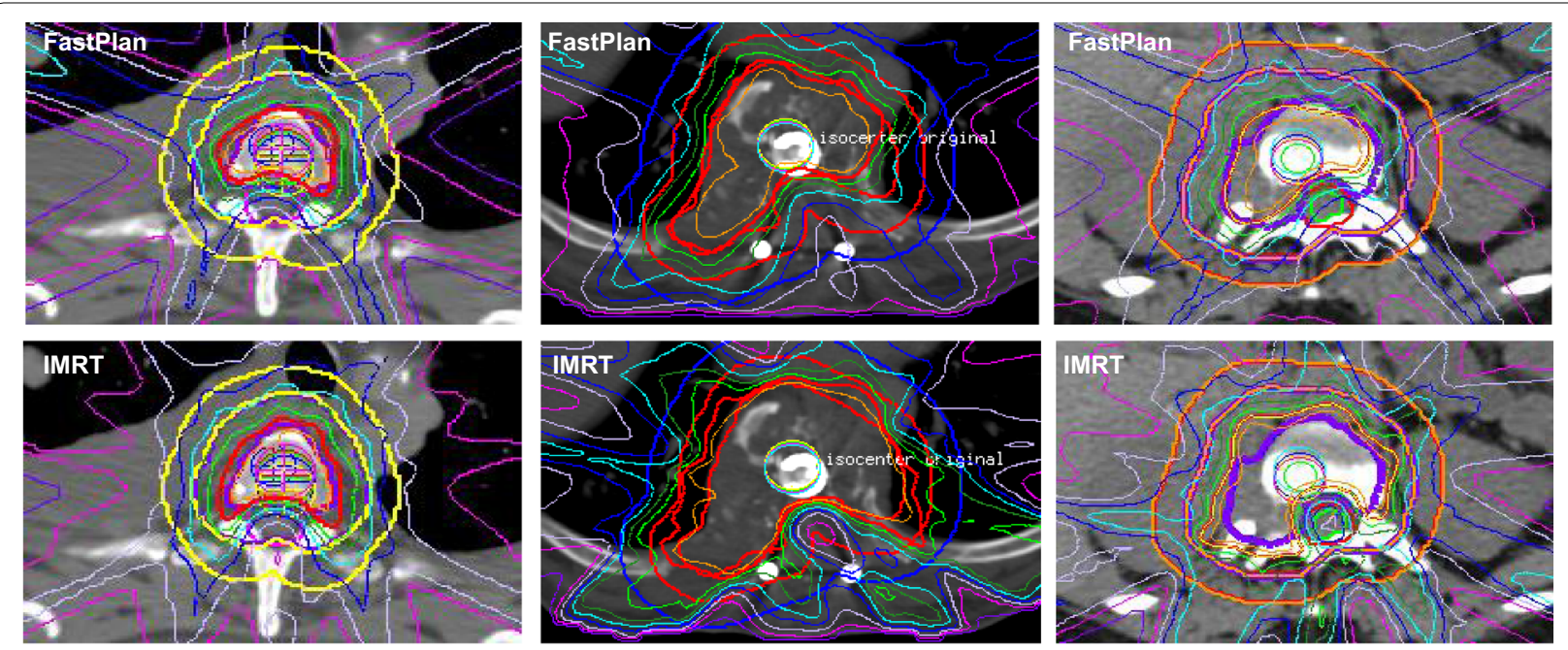

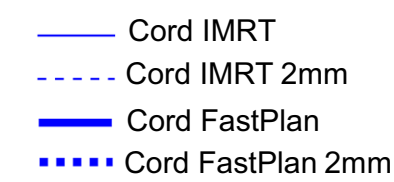

— CTV IMRT

- - - - CTV IMRT 2mm

CTV FastPlan

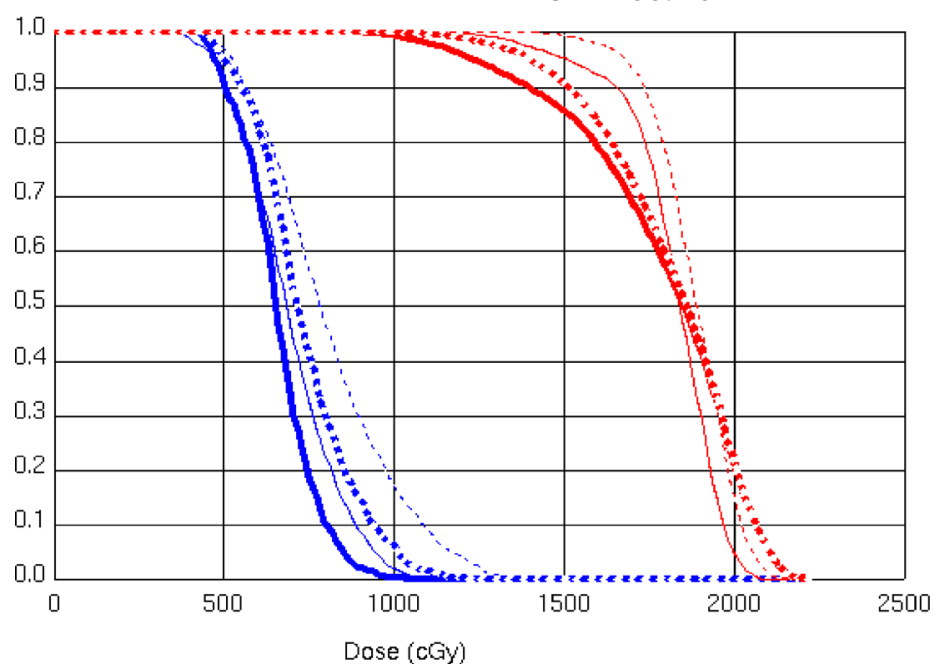

Impact of $2 \mathrm{~mm}$ Posterior Shift on Cord Dose

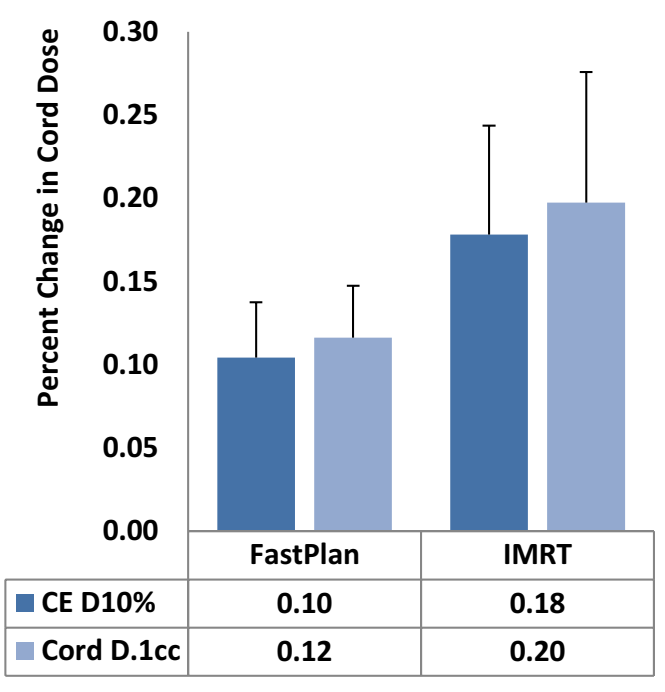

Fig. 3 Plan comparison. Top panel Representative 5-field RaSp and IMRT plans with a $2 \mathrm{~mm}$ isocenter shift to simulate setup error. Bottom Left Dosevolume histogram plots of representative cord and CTV dose for both IMRT and RaSp based plans with and without the $2 \mathrm{~mm}$ shift. Bottom Right Bar graph shows the percent change in cord dose (Cord Eval max $10 \%$ and Cord Max to $0.1 \mathrm{cc}$ ) following the 2 mm AP shift. Bars represent mean percent change for all 14 lesions; error bars show $95 \% \mathrm{Cl}$

spinal cord remained below 14 Gy for a volume of $0.03 \mathrm{cc}$. Any spinal cord dose that did not meet these criteria would have been considered a major deviation in RTOG 0631 and was therefore considered unacceptable for either IMRT or RaSp plans in this study. Treatment plans were considered adequate as long as $90 \%$ of the target volume received the prescribed radiosurgery dose and, as in RTOG 0613, dose inhomogeneity within the target volume was allowed, provided high dose spillage was limited to $105 \%$ of the prescription dose outside of the PTV volume.
We compared plan conformality using an R100 \% (conformality index) as well as intermediate-dose spillage determined from D2 $\mathrm{cm}$ and R50 \% parameters. As expected IMRT plans demonstrated a trend towards increased conformality and steeper dose gradients when compared to RaSp plans, with lower R100 \% as well as lower D $2 \mathrm{~cm}$, and R50 \% respectively. These differences, however, were not statistically significant, perhaps due to insufficient power given the number of lesions in the present analysis as well as the wide variation in PTV volumes. R50 \% and D $2 \mathrm{~cm}$ 
parameters in particular are vulnerable to volume variations and guidelines typically account for this with target volume specific cut offs (e.g., RTOG 0915 lung SBRT guidelines).

A $2 \mathrm{~mm}$ posterior shift (high dose volume shifted towards the cord), was introduced in silico to simulate a small amplitude, worst case set-up error. The $2 \mathrm{~mm}$ shift resulted in a $20 \%$ (SD $10.5 \%$ ) increase in cord dose for IMRT plans and a $10 \%$ (SD $5.3 \%$ ) increase for RaSp plans on average due to the steeper dose gradient with IMRT (Fig. 4). The same $2 \mathrm{~mm}$ anterior/posterior perturbation caused 3 cord dose violations for the IMRT plans and 1 violation for corresponding RaSp plans, illustrating a potential vulnerability of increasingly dose escalated conformal plans with steep dose fall off.

\section{Discussion}

The spine is the most frequent site of skeletal metastatic disease, accounting for nearly 20,000 new cases per year in the United States (Sciubba et al. 2009). Radiotherapy remains a mainstay of treatment for spine metastases and there is mounting evidence for the utility of stereotactic radiotherapy in preoperative as well as postoperative settings (Kaloostian et al. 2014; Chawla et al. 2009; Sahgal et al. 2011). However, limitations in SBRT as described previously have lead us to develop an alternative planning process. We have explored the feasibility and utility of an automated script for the rapid generation of high quality conformal spine radiotherapy plans that could be

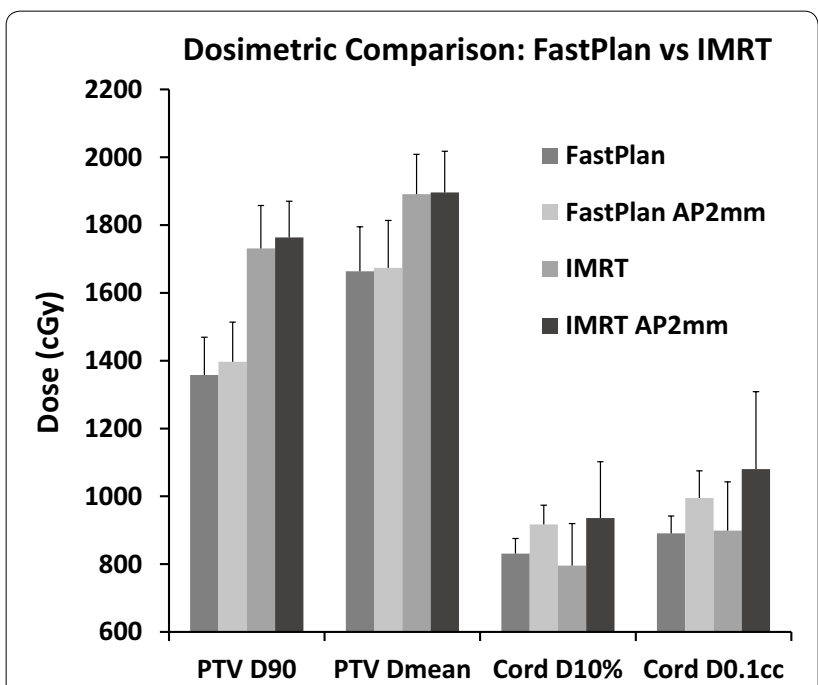

Fig. 4 RaSp vs IMRT. Average dose to $90 \%$ of the PTV (PTV D90), mean dose to PTV (PTV Dmean), maximum dose to $10 \%$ of the cord (Cord D10\%), and maximum cord dose to $0.1 \mathrm{cc}$ of the cord structure (Cord D0.1 cc) are plotted for IMRT and RaSp with and without a $2 \mathrm{~mm}$ AP shift to simulate set-up error. IMRT plans yielded more favorable dose escalation with a higher average PTV dose. A $2 \mathrm{~mm}$ shift had a greater relative impact on maximum cord dose for IMRT plans compared to RaSp plans planned and delivered with widely available resources. Here we report the preclinical validation of the "Rapid Spine" algorithm, an easily deployable and robust series of Pinnacle scripts which automate the creation of timely, dosimetrically reasonable radiotherapy plans designed to meet cooperative group standards for spine SBRT. The clinical need for such a strategy can be distilled down to two basic assumptions: (1) conformal stereotactic treatment is preferable to conventional single fraction radiotherapy for spine metastases and (2) the 'standard' IMRT workflow for stereotactic spine treatment impedes timely treatment in situations where dose escalation may otherwise be desirable.

Prospective studies have demonstrated equivalence of single fraction radiotherapy relative to fractionated regimens for pain control (Nielsen et al. 1998; Kaasa et al. 2006). Now mounting data from multiple studies have demonstrated excellent local control and durable pain relief with dose escalation above 8 Gy. Importantly, these studies have demonstrated efficacy without compromising safety, with low rates of complications such as radiation-induced myelopathy. In 2008, Ryu et al. reported on 61 previously unirradiated solitary spine metastasis treated at the Henry Ford Hospital to doses between 10 and $16 \mathrm{~Gy}$, delivered to the vertebral body and paraspinal soft tissue (Ryu et al. 2008). In this study, the median duration of pain relief was 13.6 months. One year overall survival was $74 \%$ and no cord injury was observed. Similarly, at the University of Pittsburgh, Gerszten et al. treated 393 patients with 500 spinal metastases of varying histology and including 344 previously irradiated lesions (Gerszten et al. 2007). The dose range was 12.5-25 Gy and again, no cases of radiation-induced myelopathy were reported. Long-term pain improvement was noted in $86 \%$ of patients at a median follow up of 21 months with a radiographic control rate of $75 \%$ for melanoma, $87 \%$ for renal cell carcinoma, and $100 \%$ with breast and lung carcinomas. Similar outcomes were reported by Yamada et al. at Memorial Sloan-Kettering Cancer Center (103 lesions, 93 patients, doses 18-24 Gy, no myelopathy) and Gibbs et al. at Stanford (102 lesions, 74 patients, 16-26 Gy, 3 cases of myelopathy -2 of which were in previously irradiated patients) (Yamada et al. 2008; Gibbs 2007).

Despite these and other provocative data intimating the potential superiority of stereotactic radiotherapy, few generally accepted principles exist as to the criteria for routine management of spine metastases with dose escalated radiotherapy (Hall et al. 2011; Thibault et al. 2014). The NRG has taken up this questions and the ongoing study 0631 seeks to prospectively assess improved clinical outcomes with spine SBRT using single fraction stereotactic treatment (16 Gy). The recently published phase 2 results from this study demonstrate that SRS is feasible 
without any grade $4 / 5$ treatment related toxicity for the initial 46 patients (Ryu et al. 2014).

In light of emerging data and ongoing studies, stereotactic radiotherapy has gained increasing acceptance as a primary modality in the routine management of spine metastasis. However, in its present form, IMRT-based stereotactic radiotherapy with stereotactic setup imposes logistic barriers to timely treatment in urgent and limited resource settings. Certainly, rigorous standards for treatment planning, quality assurance, and institutional credentialing are appropriate given the potential risks of SBRT (Gerszten et al. 2013). More efficient strategies are nevertheless worth exploring, as they may increase access to include patients who may benefit from dose escalation in time-sensitive cases or at institutions with limited access to resources.

The present work reports on a pilot study validating a novel approach aimed at improving efficiency through the utilization of automated treatment planning scripts. The integrated RaSp workflow consolidates the steps involved in stereotactic treatment planning, including CT based simulation, contouring, inverse treatment planning with limited beam segmentation, and finally, standard calculation based verification (Fig. 1). The RaSp treatment planning algorithm is customizable and relies at its core on a risk adapted approach, limiting the final prescription by dose to the spinal cord. The simulation parameters as well as physician contouring and delineation of target and OAR structures are identical for both RaSp and standard approaches.

Automated RaSp plans were generated quickly (on the order of minutes) after target and OAR structures were defined and yielded very reasonable dosimetry including a mean dose to the PTV of 1663 cGy after limiting dose to the spinal cord to RTOG 0631 limits. As expected, $\mathrm{RaSp}$ plans were less conformal than corresponding IMRT plans as reflected in a trend towards higher R100 \%. The increased beam number and segmentation utilized for IMRT plans (necessitating more labor-intensive IMRT QA for verification) resulted in a steeper dose gradient at the PTV/Cord interface, as well as a trend towards lower D $2 \mathrm{~cm}$ and R50 \% intermediate dose spillage, allowing more favorable dose escalation with a mean dose to the PTV of $1891 \mathrm{cGy}-13$ \% higher than corresponding RaSp plans on average. Target coverage was similarly improved with IMRT with a $22 \%$ increase in PTV D90 over corresponding RaSp plans, on average.

The steeper dose gradient observed with IMRT led us to hypothesize that IMRT-based plans may be more sensitive to setup error. Indeed we found that a $2 \mathrm{~mm}$ anterior/posterior shift in the isocenter resulted in a $20 \%$ (SD $10.5 \%$ ) increase in cord dose for IMRT plans and a $10 \%$ (SD $5.3 \%$ ) increase for RaSp plans on average. The same $2 \mathrm{~mm}$ perturbation caused 3 cord dose violations for
IMRT plans and only 1 violation for corresponding RaSp plans. The smaller dosimetric impact of setup error suggests that appropriate safety may be maintained even during urgent therapy of patients in distress. Furthermore, in urgent situations where surgery may be preferable to SBRT, for example in cases of extensive epidural extension of disease (Patchell et al. 2005; Sahgal et al. 2008; Laufer et al. 2013), RaSp may offer an alternative approach for safe dose escalation in persons otherwise ineligible for surgical decompression. While further investigation is required to improve the process proposed here, the prospect of providing a safer and faster option for dose escalation radiation treatment to a broader spectrum of patients nationally and worldwide is intriguing.

On the whole, the RaSp algorithm efficiently and robustly yielded dosimetrically reasonable plans when compared to more resource-intensive IMRT-based spine radiotherapy. When feasible, more complex planning (e.g. IMRT, CyberKnife Multiplan) is preferable given its increased conformality and the possibility of increased dose escalation. However, in urgent situations, rapid initiation of treatment is particularly important, especially where invasive decompression is contraindicated. Speed of treatment initiation may be of importance in cases of symptomatic and asymptomatic cord compression, and thus may be a value to balance against the potential benefit of further dosimetric optimization. Also, in limited resource situations where SBRT may be unavailable, patients may benefit from a safe, time efficient, and robust method for planning dose escalated single fraction radiotherapy.

Implementations strategies for the clinical utilization of RaSp scripting are in development with a focus on optimizing more complex beam geometries yielding more favorable conformality, publishing open-source scripts for multiple treatment planning platforms, and prospective validation in early phase prospective clinical trials. With the use of automated scripts, the marginal reduction in conformality must be weighed against the benefit of expeditious treatment. Such tradeoffs are appropriately made in other clinical circumstances varying from urgent radiation techniques to emergent invasive surgeries, and prospective clinical validation with RaSp is the necessary next step in setting a standard for the ideal balance between time and conformality. The Rapid Spine approach reduces the tradeoff between rapid treatment and optimal dosimetry in urgent situations, warranting further development and clinical validation of this approach.

\section{Abbreviations}

CGy: centigray; CTV: clinical target volume; Dmax: maximum point dose; Gy: gray; IMRT: intensity-modulated radiation therapy; PTV: planning target volume; QA: quality assurance; RaSp: Rapid Spine; SBRT: stereotactic body radiation therapy; SD: standard deviation. 


\section{Authors' contributions}

Dr. OM, Dr. OT, Dr. LK initiated the study, analyzed the data, and developed the RaSp algorithm. Joy Lee contributed to the writing and submission of the manuscript. Dr. YL and Dr. TM contributed to analysis of the data, wrote the Pinnacle scripts, and generated treatment plans for comparison. Dr. ML, Dr. DR, Dr. J-P W, Dr. DS, Dr. ZG, and Dr. KR jointly selected treated spine metastases for inclusion from the Hopkins spine surgery/radiosurgery database, evaluated stereotactic treatment plans, and revised the manuscript. All authors read and approved the final manuscript.

\section{Author details}

${ }^{1}$ Department of Radiation Oncology, Cleveland Clinic, Taussig Cancer Institute, Cleveland, OH, USA. ${ }^{2}$ Delmarva Radiation Services, Tunnell Cancer Center, Rehoboth Beach, DE, USA. ${ }^{3}$ Department of Radiation Oncology and Molecular Radiation Sciences, Sidney Kimmel Comprehensive Cancer Center, The Johns Hopkins University School of Medicine, Baltimore, MD, USA. ${ }^{4}$ Department of Radiation Oncology, Indiana University, Indianapolis, IN, USA. ${ }^{5}$ Department of Neurosurgery, The Johns Hopkins University School of Medicine, Baltimore, MD, USA.

\section{Acknowledgements}

The authors would like to acknowledge Juan Jackson, CMD and Ted Hooker, CMD for dosimetric planning consultation and for providing insights into inverse planning methodology used for stereotactic treatments.

\section{Competing interests}

Omar Y. Mian, Owen Thomas, Joy JY Lee, Yi Le, Michael Lim, Daniele Rigamonti, Jean-Paul Wolinsky, Ziya L. Gokaslan, and Lawrence Kleinberg declare that they have no competing interests. Todd McNutt conducts research on shape-based automated treatment planning in collaboration with Philips Radiation Oncology Systems. Daniel M. Sciubba consults for Medtronic and receives honoraria from Depuy Synthes, Globus, and Orthofix. Kristin Redmond is the recipient of a research grant from Elekta $A B$.

\section{Compliance with ethical standards}

Statement of informed consent was not applicable since the manuscript does not contain any patient data. No human or animal studies were conducted, exempting this study from IRB/IACUC approval requirements for human subjects or animal research respectively, as stated in institutional guidelines. All procedures followed were in accordance with the ethical standards of the responsible committee on human experimentation (institutional and national) and with the Helsinki Declaration of 1975, as revised in 2008.

Received: 5 March 2016 Accepted: 29 July 2016

Published online: 11 August 2016

\section{References}

Amdur RJ, Bennett J, Olivier K et al (2009) A prospective, phase II study demonstrating the potential value and limitation of radiosurgery for spine metastases. Am J Clin Oncol 32(5):515-520

Chawla S, Abu-Aita R, Philip A, Lundquist T, Okunieff P, Milano MT (2009) Stereotactic radiosurgery for spinal metastases: case report and review of treatment options. Bone 45(4):817-821

Cox BW, Spratt DE, Lovelock M et al (2012) International spine radiosurgery consortium consensus guidelines for target volume definition in spinal stereotactic radiosurgery. Int J Radiat Oncol Biol Phys 83(5):e597-e605

Gerszten PC, Burton SA, Ozhasoglu C, Welch WC (2007a) Radiosurgery for spinal metastases: clinical experience in 500 cases from a single institution. Spine (Phila Pa 1976) 32(2):193-199
Gerszten PC, Burton SA, Ozhasoglu C, Welch WC (2007b) Radiosurgery for spinal metastases: clinical experience in 500 cases from a single institution. Spine (Phila Pa 1976) 32(2):193-199

Gerszten PC, Sahgal A, Sheehan JP et al (2013) A multi-national report on methods for institutional credentialing for spine radiosurgery. Radiat Oncol 8:158-717X-8-158

Gibbs IC (2007) Spinal and paraspinal lesions: the role of stereotactic body radiotherapy. Front Radiat Ther Oncol 40:407-414

Hall WA, Stapleford LJ, Hadjipanayis CG, Curran WJ, Crocker I, Shu HK (2011) Stereotactic body radiosurgery for spinal metastatic disease: an evidencebased review. Int J Surg Oncol. doi:10.1155/2011/979214

Hartsell WF, Scott CB, Bruner DW et al (2005) Randomized trial of short- versus long-course radiotherapy for palliation of painful bone metastases. J Natl Cancer Inst 97(11):798-804

Howell DD, James JL, Hartsell WF et al (2013) Single-fraction radiotherapy versus multifraction radiotherapy for palliation of painful vertebral bone metastases-equivalent efficacy, less toxicity, more convenient: a subset analysis of radiation therapy oncology group trial 97-14. Cancer 119(4):888-896

Kaasa S, Brenne E, Lund JA et al (2006) Prospective randomised multicenter trial on single fraction radiotherapy $(8 \mathrm{~Gy} \times 1$ ) versus multiple fractions (3 Gy $\times 10$ ) in the treatment of painful bone metastases. Radiother Oncol 79(3):278-284

Kaloostian PE, Yurter A, Zadnik PL, Sciubba DM, Gokaslan ZL (2014) Current paradigms for metastatic spinal disease: an evidence-based review. Ann Surg Oncol 21(1):248-262

Laufer I, lorgulescu JB, Chapman T et al (2013a) Local disease control for spinal metastases following "separation surgery" and adjuvant hypofractionated or high-dose single-fraction stereotactic radiosurgery: outcome analysis in 186 patients. J Neurosurg Spine 18(3):207-214

Laufer I, Rubin DG, Lis E et al (2013b) The NOMS framework: approach to the treatment of spinal metastatic tumors. Oncologist 18(6):744-751

Lo SS, Lutz ST et al (2013) ACR appropriateness criteria (R) spinal bone metastases. J Palliat Med 16(1):9-19

Nielsen OS, Bentzen SM, Sandberg E, Gadeberg CC, Timothy AR (1998) Randomized trial of single dose versus fractionated palliative radiotherapy of bone metastases. Radiother Oncol 47(3):233-240

Patchell RA, Tibbs PA, Regine WF et al (2005) Direct decompressive surgical resection in the treatment of spinal cord compression caused by metastatic cancer: a randomised trial. Lancet 366(9486):643-648

Ryu S, Jin R, Jin JY et al (2008) Pain control by image-guided radiosurgery for solitary spinal metastasis. J Pain Symptom Manage 35(3):292-298

Ryu S, Pugh SL, Gerszten PC et al (2011) RTOG 0631 phase II/III study of imageguided stereotactic radiosurgery for localized (1-3) spine metastases: phase II results. Int J Radiat Oncol Biol Phys 81(2):S131-S132

Ryu S, Pugh SL, Gerszten PC et al (2014) RTOG 0631 phase 2/3 study of image guided stereotactic radiosurgery for localized (1-3) spine metastases: phase 2 results. Pract Radiat Oncol 4(2):76-81

Sahgal A, Larson DA, Chang EL (2008) Stereotactic body radiosurgery for spinal metastases: a critical review. Int J Radiat Oncol Biol Phys 71(3):652-665

Sahgal A, Bilsky M, Chang EL et al (2011) Stereotactic body radiotherapy for spinal metastases: current status, with a focus on its application in the postoperative patient. J Neurosurg Spine 14(2):151-166

Schipani S, Wen W, Jin JY, Kim JK, Ryu S (2012) Spine radiosurgery: a dosimetric analysis in 124 patients who received 18 Gy. Int J Radiat Oncol Biol Phys 84(5):e571-e576

Sciubba DM, Nguyen T, Gokaslan ZL (2009) Solitary vertebral metastasis. Orthop Clin North Am 40(1):145-54, viii

Thibault I, Al-Omair A, Masucci GL et al (2014) Spine stereotactic body radiotherapy for renal cell cancer spinal metastases: analysis of outcomes and risk of vertebral compression fracture. J Neurosurg Spine 21(5):711-718

Yamada Y, Bilsky MH, Lovelock DM et al (2008) High-dose, single-fraction image-guided intensity-modulated radiotherapy for metastatic spinal lesions. Int J Radiat Oncol Biol Phys 71(2):484-490 